\title{
Research on Support Vector Machine in Image Denoising
}

\author{
Xinfeng Guo and Chunyan Meng \\ Computer Engineering of Shanxi University \\ Taiyuan, China \\ E-mail:tydzgxf@126.com \\ Center of Computer, Taiyuan Normal University \\ Taiyuan, China \\ E-mail:lvshuyang@163.com
}

\begin{abstract}
In this paper, a denoising algorithm and simulation experiments of algorithm based on wavelet transform and support vector machine (SVM) image is proposed, a new method is adopted in the selection of characteristic vector of support vector machine, based on training of support vector machine, the support vector machine model is used to distinguish between noise and the original image, to achieve the effect of denoising. The experimental results show that the method can well remove the noise, and can save some important details of images, compared with other denoising method based on wavelet transform, it has a good advantage.
\end{abstract}

Keywords: Wavelet transforms, Support vector machine, Characteristic vector, Image denoising

\section{Introduction}

With the continuous development of social informatization and the enhanced ability of the computer processing, people now have entered the information era of life, images and more and more closely related to people's life and production, has been applied to the digital television, television, telephone, military, aerospace, medical, industrial, agriculture and other aspects. However, due to the image in the process of acquisition, compression, conversion and transmission, to avoid the moderating effect of their own equipment and a variety of external factors, to produce some unnecessary noise, such as electronic noise, speckle noise, the photon noise and quantization noise. So most of the real image with noise, if the signal-to-noise ratio is too low, can affect the quality of the image, which caused a big trouble to people's actual needs. In order to improve the image quality and quality, provide convenience to people's production and life, image denoising has become a hot issue study in various fields. A good denoising method is in does not affect the image under the premise of important details, as much as possible to remove noise.

In the field of image denoising, people are given the multi-resolution characteristic of wavelet transform is widely adopted; the denoising method based on wavelet coefficient is the researchers usually adopt algorithm. Image denoising in a certain sense is to separate the noise and the image process, the actual is a classification problem. Support vector machine as a good classifier, gradually received widespread attention in the field of image processing. Such as the United States postal handwritten digital library recognition, face detection, validation, denoising, image segmentation, and involves the theory of support vector machine and got a great success. This chapter puts forward a kind of based on wavelet transform and support vector machine de-noising algorithm, the experiment results show that the method can achieve higher peak signal to noise ratio, has the very good denoising effect. 
Support vector machine is a Vapnik8, et al., first proposed in 1995, because of the support vector machine is suitable for dealing with small sample, high dimension and better generalization performance and so on many aspects of advantages, is widely used in the classification problem. Support vector machine method is based on VC dimension theory and structure risk minimum principle basis, we can calculate it for support vector classification and better ability to distinguish, and probably in support vector attachment midperpendicular direction is the position of the optimal hyperplane. Hyperplane can maximize classification intervals to ensure the accuracy of classification and minimum classification error.

Suykens proposes new support vector machine types of least squares support vector machine (SVM). It USES solving a system of linear equations instead of the traditional support vector machine USES the method of quadratic programming, simplifies the complexity of optimization problem in algorithm. 1998 Platt realizes serialization of support vector machine, for the classification problem and pattern recognition has important significance. Compared with the traditional neural network, support vector machine has simple structure design, obviously improve the generalization ability, in pattern recognition, signal processing, system modeling and optimal control in the areas of application is very extensive.

\section{Related Works}

Support vector machine (SVM) in the aspect of image denoising has been very widely used, for example: Wang Shunli presents an image denoising method based on support vector machine (SVM), the method using support vector regression technology structure filter required for image denoising; One of the feature extraction and the design of the training sample aimed at curbing the different types of noise. FuYan proposed a weighted SVM based on feature combination and image denoising methods, according to the correlation of adjacent pixels in the image and the characteristics of the salt and pepper noise, extract contains a variety of noise in the image characteristics. Zhang Xuegong put forward at the center of the Support vector machine (Central Support VectorMachines, CSVM) method, each type of training sample set clustering is divided into several subsets, center with a subset of the new training sample set training SVM; Keerthi modification method put forward by the NPA (Nearly PointAlgorithm) recently points method, to a certain extent, improve the convergence speed of the proposed method. Based on support vector machine (SVM) the singular point of capture performance, Ma Xueliang will support vector regression model generation support vector filter and the sampling direction filter bank, is applied to the remote sensing in the SAR image denoising.

The traditional statistics is by gradual theory to explore the samples tends to infinite time's characteristics; explore the joint degree of mathematical models and assumptions. Statistical method processing mainly can be divided into three stages: data collection, data analysis, reasoning, classification and prediction. Common statistical methods include regression analysis, cluster analysis, exploratory analysis and discriminate analysis

Support vector machine (SVM) is based on VC dimension theory and structural risk minimization principle. Its main idea is to the problem, two kinds of classification is mapped to high-dimensional space to seek a hyperplane as segmentation of two types of problems, to ensure the minimum classification error rate. Support vector machine (SVM) to handle the linearly separable case and the linear inseparable, classification is a very practical tool.

Support vector machine (SVM) is based on statistical theory arises at the historic moment, it is based on $\mathrm{VC}$ dimension theory and structural risk minimization principle, independent found beneficial to support vector classification, so as to construct the classification hyperplane. Support vector machine (SVM) by learning to find the optimal hyperplane, has the largest classification interval. The basic idea of SVM is by using 
kernel function to low-dimensional input space data mapped to high-dimensional feature space, the low dimensional linear inseparable problem under the high dimensional space become linearly separable.

Empirical risk minimum principle of consistency can be described as: the function set $L(\mathrm{~m}, \mathrm{n})$ and probability distribution function $\mathrm{F}(\mathrm{x}, \mathrm{y})$, in the case of infinitely many samples if can converge to the same limit value, said the empirical risk minimum consistency. And the actual risk and empirical risk with at least $1-\eta$ probability to satisfy the following formula ${ }^{[3]}$ :

(1)

$$
R(w) \leq R_{e m p}(w)+\sqrt{\frac{h(\ln (2 l / h+1)-\ln (\eta / 4))}{l}}
$$

Among them, $l$ is sample, $h$ is A set of functions of VC dimension, $0 \leq \eta \leq 1$, actual risk is composed of empirical risk and confidence limit, confidence limit to reflect the real risk and the upper bound of the empirical risk difference, it is associated with the VC peacekeeping sample of function sets, it gets bigger and bigger with $\mathrm{VC}$ dimension $h$ smaller with sample sizes $l$.

Structural risk minimization generally there are two ways: first, calculate each subset of the empirical risk minimum, and then select the empirical risk and confidence interval is the smallest; Second, the constructor sets the empirical risk minimum, and then select the confidence interval, the smallest (support vector machine is based on the second thought). Structural risk minimization principle is in the case of limited samples, have found empirical risk and confidence interval, this approach can achieve the ideal.

\section{Image Denoising Process Design}

In the field of image denoising, people are given the multi-resolution characteristic of wavelet transform is widely adopted, which is based on the wavelet coefficient denoising method ${ }^{[1]}$ is the researchers usually adopt the algorithm. For an image, the edge and contour and texture are mainly concentrated on the high frequency information, describe the detail of the image changes, and the noise is due to the randomness, is usually included in the high frequency information. Therefore, at the same time of denoising is bound to be detrimental to details, to be a good reservation details are difficult to achieve the ideal denoising. How to do to find an ideal compromise balance is crucial. Wavelet threshold denoising method is applied in the traditional wavelet transform denoising most widely, and its basic ideas are as follows: (1) the image for discrete wavelet decomposition, get the high frequency subband and low-frequency subband and wavelet coefficients of each direction. (2) Threshold. Determine the threshold for parties to the wavelet coefficient threshold upward. (3) Using the modified coefficient of inverse wavelet image for image reconstruction。

Some scholars in the training process for support vector machine optimization problem, puts forward some improvement of genetic algorithm and neural network algorithm combining with algorithm, greatly improving the efficiency of support vector machine, save the training time. With existing machine learning, such as neural network, fuzzy compared such as machine learning, artificial intelligence, genetic method, support vector machine has a more solid theoretical foundation and better generalization ability and strong nonlinear and high dimensional processing ability. Can be expected, in quite a long time in the future, about this aspect of the further research will bring about a sustainable development boom.

In this process, the selection of threshold is suitable is the most key factors influencing the denoising effect is good or bad. Threshold selection is too small, will keep too much noise, cannot achieve the expected effect of denoising; the coefficient of threshold selection is too large, can make the image too much into 0, damaged the image blur. 
Generally on the choice of the threshold, there are two major categories: ${ }^{[2]}$ soft threshold and hard threshold. Hard threshold denoising is easy to cause the image edges mutations, method of soft threshold denoising images sometimes too smooth. The traditional threshold denoising method, large size threshold is given, the denoising effect is often certain subjectivity and randomness, in many cases, can't get a satisfactory result.

Support vector machine as a new classification algorithm, was widely used in many fields. Its algorithm in image denoising process is: to contain noise image wavelet decomposition, and then select eigenvectors, training and support vector machine classification, finally the image reconstruction.

(1) Wavelet decomposition ${ }^{[4,5]}$ : the noise image $\mathrm{J}$ levels discrete wavelet transform, get A low-frequency subband A and high frequency subband $D_{j}, j=1,2, \sim J$, and the wavelet coefficients $Y(i, j)$ of different scale and direction, as shown in the figure below:

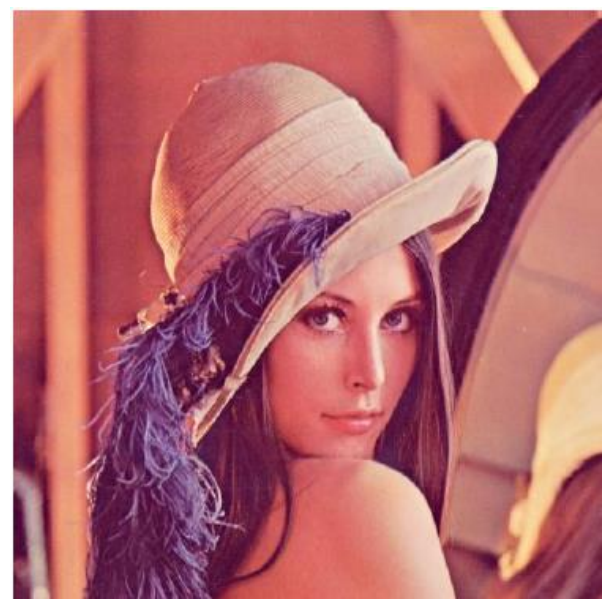

Lena image

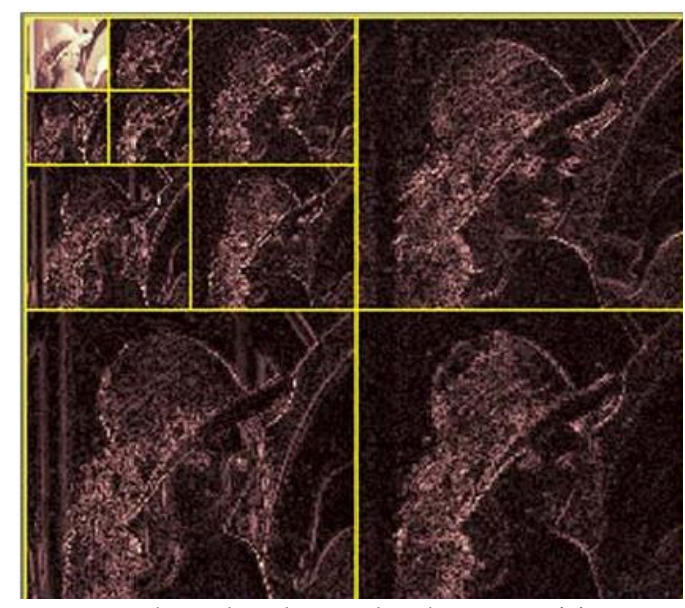

three-level wavelet decomposition

\section{Figure 1. Wavelet Decomposition}

In the upper left corner for each level of the approximate part, i.e., the low frequency component, the upper right corner to horizontal component, the lower left corner as the vertical component, the lower right corner of the diagonal components. The main part of the image for the low frequency part, reason of low frequency part decomposition step by step, and get rid of the rest of the high frequency part is the image multistage decomposition (2). Select eigenvectors: for support vector machine classification process, the characteristic vector selection of a great influence on classification results. By using properties of the wavelet coefficient, use neighborhood wavelet coefficients of the average amplitude values to do select eigenvectors as an important reference basis. First of all, will get the wavelet coefficient after wavelet decomposition as below for processing ${ }^{[6]}$ :

$$
X(\mathrm{i}, \mathrm{j})=\left\{\begin{array}{l}
1,|Y(i, j)| \geq T_{j} \\
0,|Y(i, j)|<T_{j}
\end{array}\right.
$$

Among them $T_{j}$ is as the threshold value of the series,

$$
T_{j}=a \times \sqrt{2 \ln N} / e^{\frac{j-1}{j+1}}, j=1,2, \sim, J
$$

Among them, $a=\frac{\text { median }\left(\left|Y_{i}(i, j)\right|\right)}{0.6745}, Y_{i}(i, j) \in D_{i}$. 
In the high frequency part $D_{j}, j=1,2, \sim J$ of image, if the window dimension of $2 \mathrm{n}+$ 1 , this window corresponds to the neighborhood center wavelet coefficients of the average amplitude value ${ }^{[7]}$ is:

$$
A(i, j)=\frac{\sum_{l=-n}^{n} \sum_{m=-n}^{n}\left|X_{k}(i+l, j+m)\right|-\left|X_{k}(i, j)\right|}{(2 n+1)^{2}-1}
$$

(4)

Then the feature vector is:

$$
S=\left\{\begin{array}{l}
S_{k}^{1}, A(i, j) \geq A \\
S_{k}^{2}, A(i, j)<A
\end{array}\right.
$$

(3) Support vector machine training: support vector machine has many characteristics, one of them is raising dimension for data processing, support vector machine parameters and the dimension of feature space there is no direct relationship. Raising dimension here is implemented by kernel function, different kernel function to map the characteristics of the different space, the index of different feature space is unable to compare. Minimize cross validation method is used to determine the parameters for the kernel function in this paper, the selection of kernel function for radial basis kernel function, as shown below ${ }^{[8,}$ 9]:

$$
K\left(x, x_{i}\right)=\exp \left\{-\frac{\left\|x-x_{i}\right\|^{2}}{a^{2}}\right\}
$$

(6)

Each of which corresponds to a support vector function center, they all be determined by the algorithm and its output value.

(4) Support vector machine classification: use the trained support vector machine to classify the wavelet coefficients of high frequency subband. All of the high frequency coefficients will be divided into two categories: noise coefficient and the noise coefficient. When the support vector machine when the output is 1 , high frequency coefficient is the noise factor; when the output of the support vector machine when the result is 1 , high frequency coefficient represents the noise coefficient.

(5) Image reconstruction: the noise coefficient follows below equation for soft threshold processing ${ }^{[10]}$ :

(7)

$$
W_{i j}^{k}=\left\{\begin{array}{l}
\operatorname{sign}\left(w_{i j}^{k}\right)\left(\left|w_{i j}^{k}\right|-T\right),\left|w_{i j}^{k}\right| \geq T \\
0,\left|w_{i j}^{k}\right|<T
\end{array}\right.
$$

Among them $W_{i j}^{k}$ is wavelet coefficient after soft threshold processing, $w_{i j}^{k}$ is the wavelet coefficient before processing, $T$ as the threshold value, the change with classification number according to (3), using wavelet coefficients obtained by inverse wavelet reconstruction after denoising images can be obtained.

\section{Experiment and Analysis}

The original image, each House and Lena adding different levels of gauss noise and salt and pepper noise, and then the method in this paper made a comparison and reference method [14]. Gives a denoising method based on wavelet coefficient relation, it is compared with the traditional threshold denoising method has higher peak signal to noise 
ratio. Image denoising effect evaluation standard of quality we adopt common peak signal-to-noise ratio, which are defined as follows ${ }^{[11,12 \text {, and 13]: }}$

$$
M=\frac{1}{\mathrm{~m} \times \mathrm{n}} \sum_{i=1}^{m} \sum_{j=1}^{n}\left(\mathrm{f}(\mathrm{i}, \mathrm{j})-N(\mathrm{i}, \mathrm{j})-X^{\prime}(\mathrm{i}, \mathrm{j})\right)^{2}
$$

(8)

$$
P=-10 \lg \frac{M}{255^{2}}
$$

(9)

Among them, $M$ is variance, $P$ is peak signal to noise ratio, $\mathrm{f}(\mathrm{i}, \mathrm{j})$ is original image signals with noise, $X^{\prime}(\mathrm{i}, \mathrm{j})$ is estimated image after denoising, $N(\mathrm{i}, \mathrm{j})$ is the estimated noise, $\mathrm{m} \times \mathrm{n}$ is the size of image. Below in House image and Lena image, for example, shows the noise level of 0.05 denoising effect with the method of wavelet transform and our way for comparison, for the comparison of corresponding $\mathrm{P}$ values in Table 1.

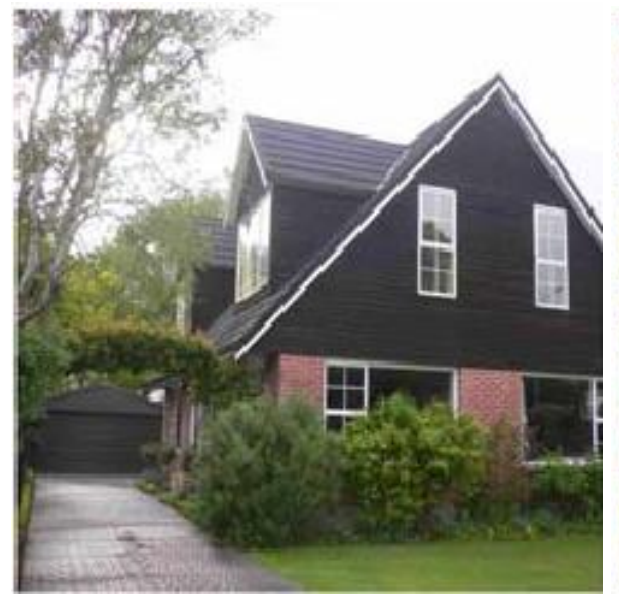

House image

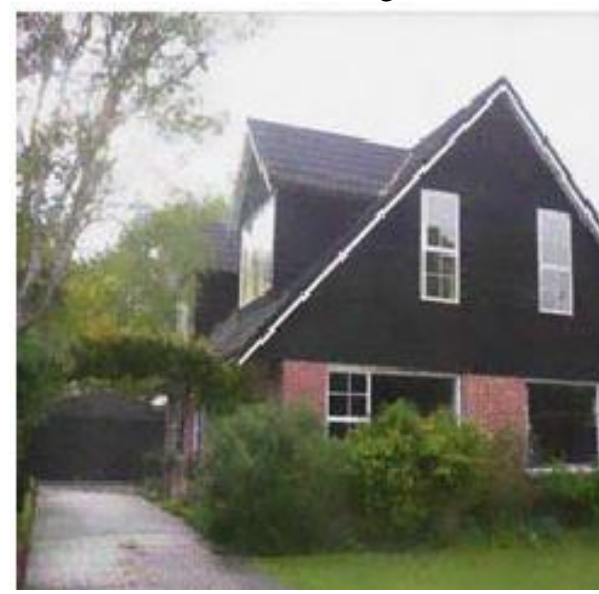

Wavelet denoising

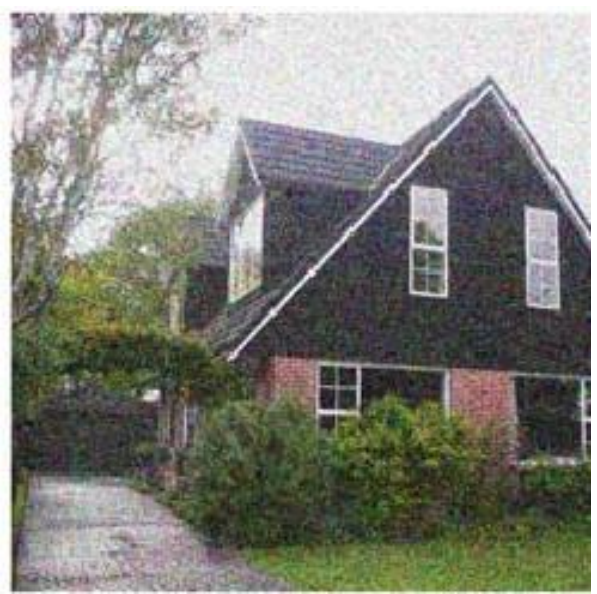

Gauss noise

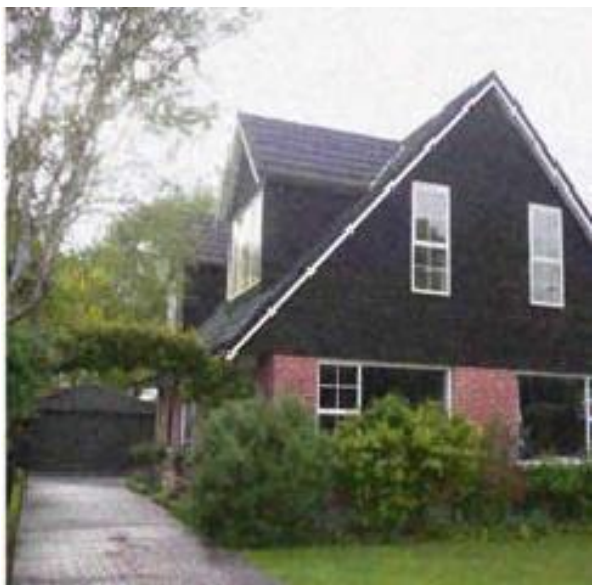

Our way 


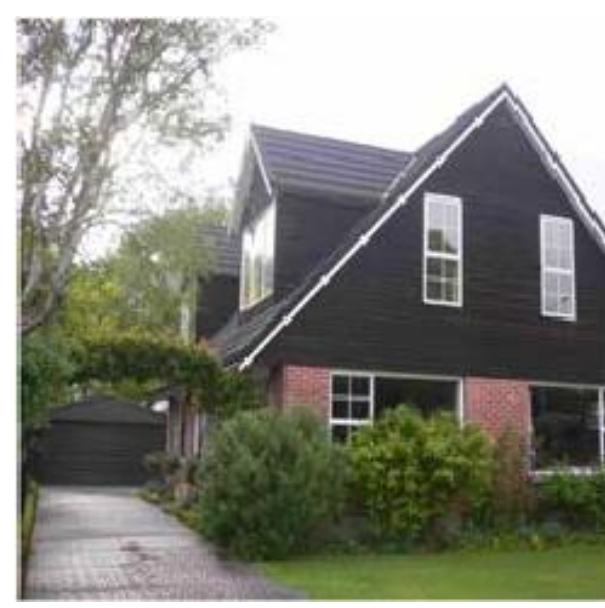

House image

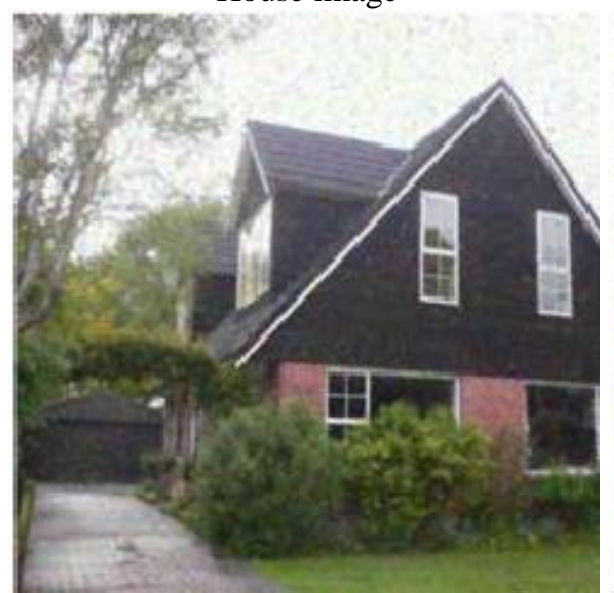

Wavelet denoising

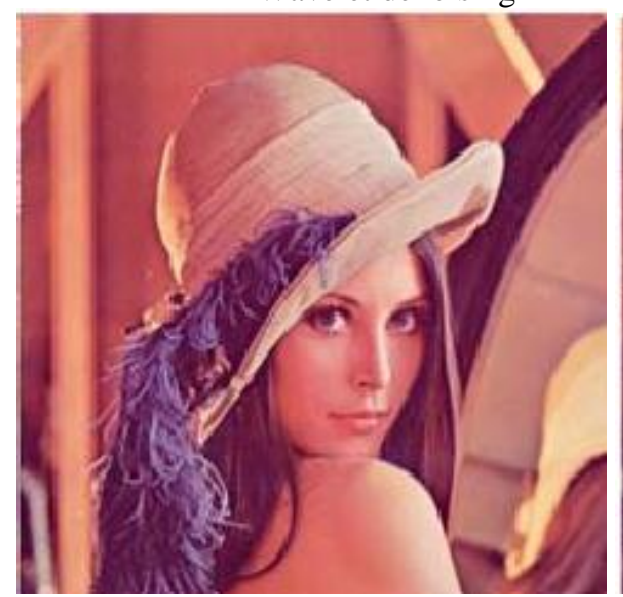

Lena image

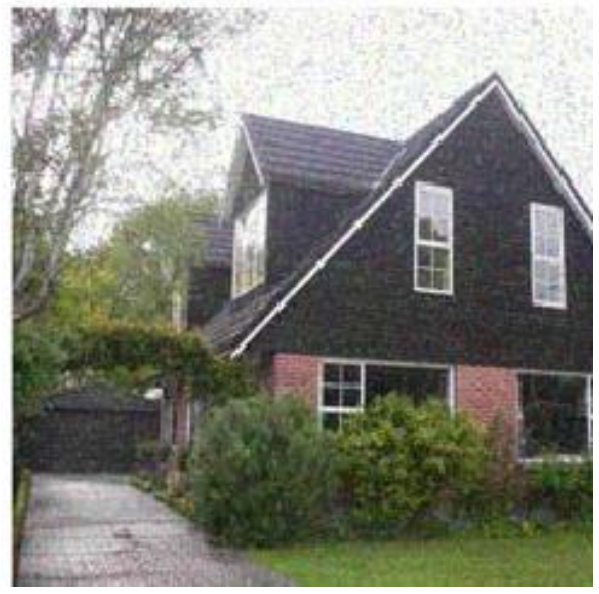

Impulse noise

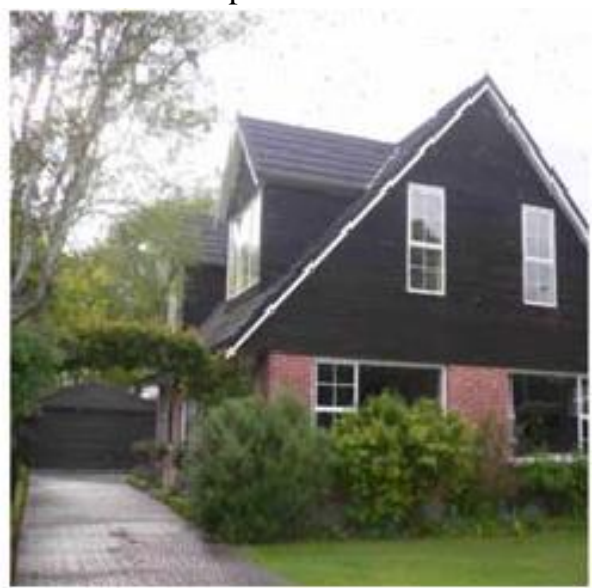

Our way

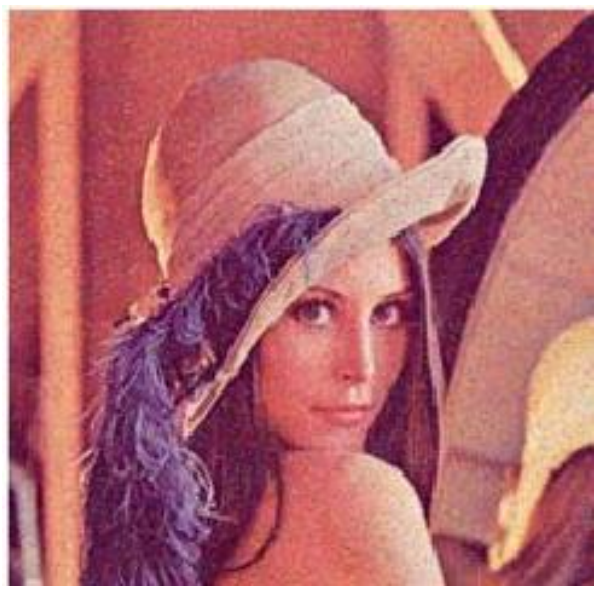

Gauss noise 

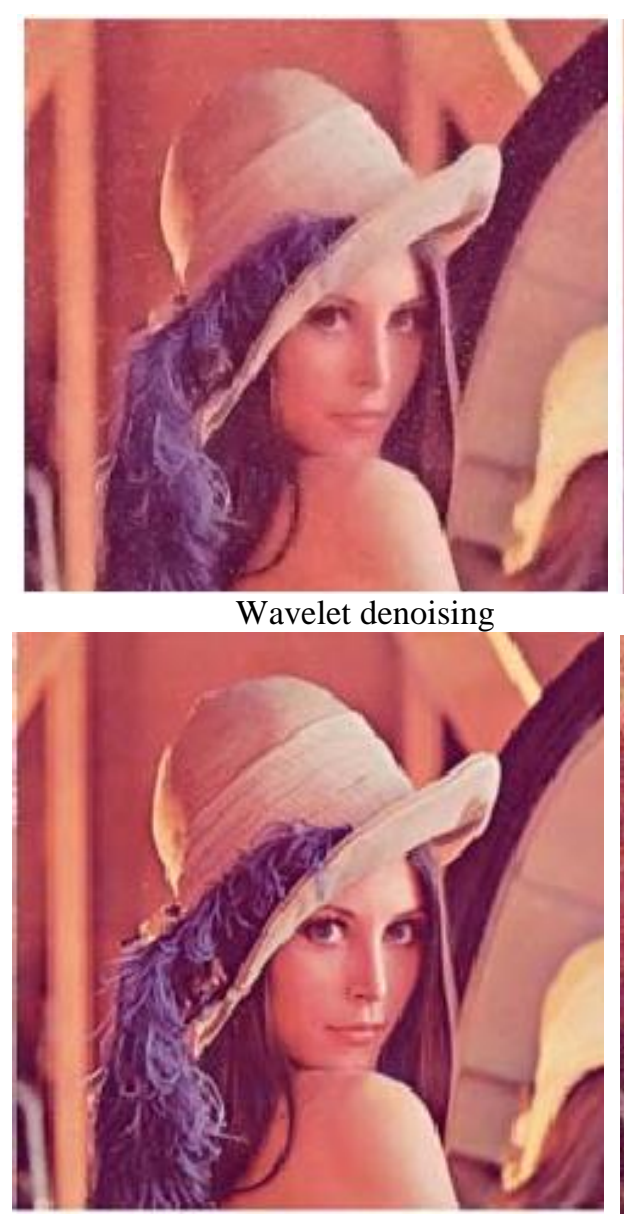

Lena image

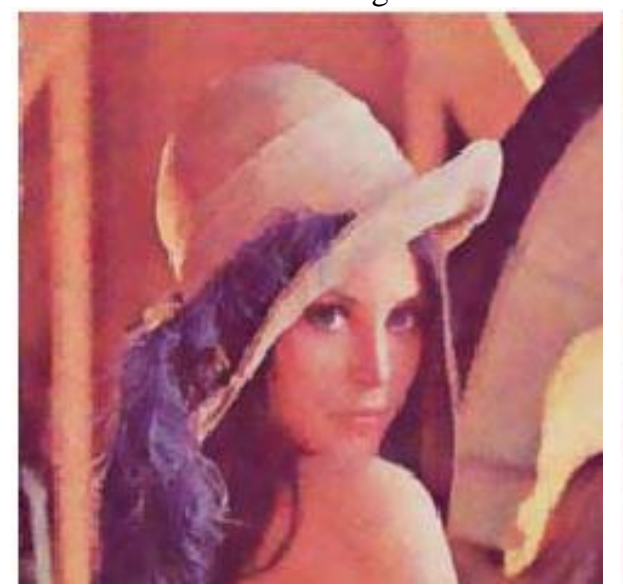

Wavelet denoising

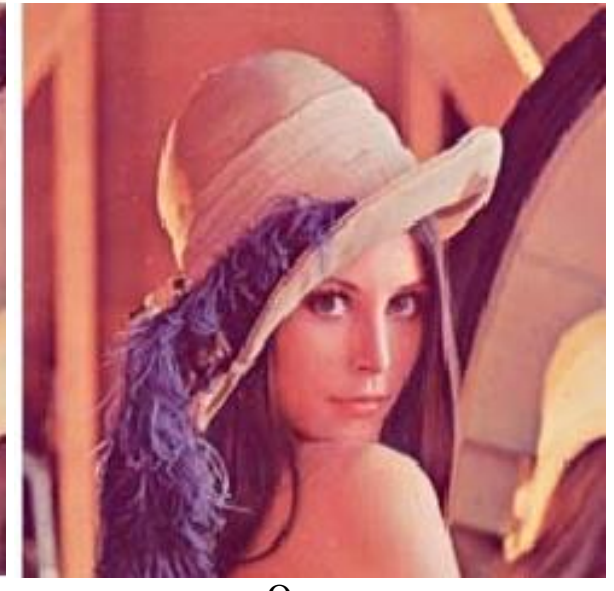

Our way

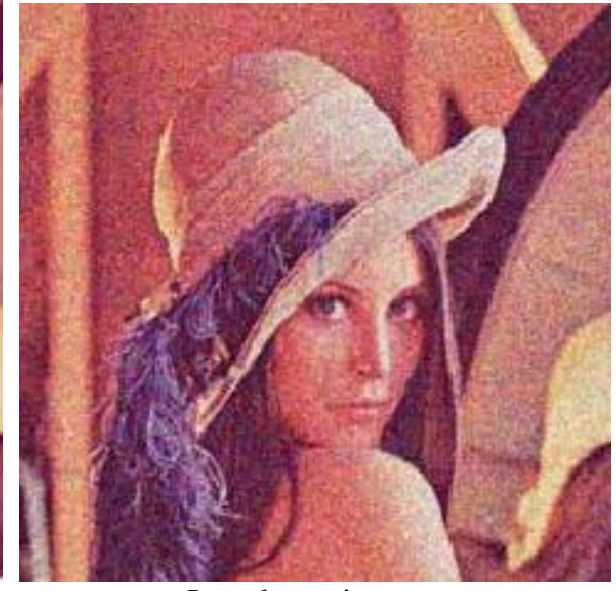

Impulse noise

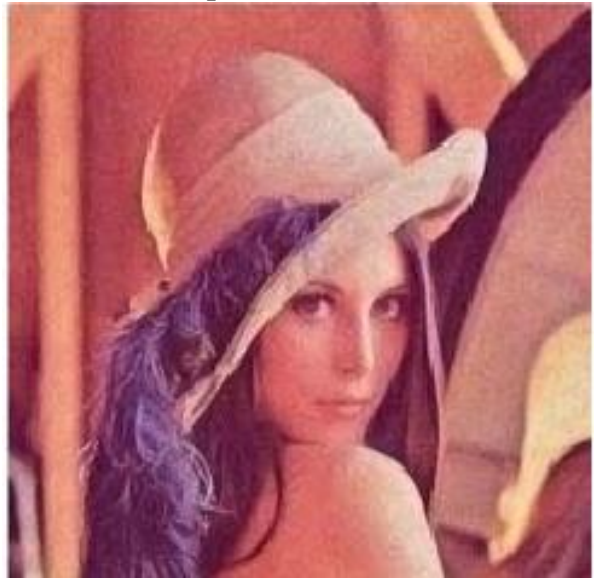

Our way

Figure 2. Denoising Effect Comparison

Table 1. Comparison of the Peak Signal to Noise Ratio (db)

\begin{tabular}{|c|c|c|c|}
\hline Image & Noise & Wavelet denoising & Our way \\
\hline \multirow{2}{*}{ House } & Gaoss noise & 39.7 & 40.5 \\
\cline { 2 - 4 } & Impulse noise & 31.4 & 32.9 \\
\hline \multirow{2}{*}{ Lena } & Gaoss noise & 37.2 & 38.8 \\
\cline { 2 - 4 } & Impulse noise & 31.5 & 33.3 \\
\hline
\end{tabular}


Can be seen clearly from Figure 3.10, in this paper, the method can make it as far as possible at the same time in the denoising of preserved the original image is one of the important details, such as Lena hats, hair and shoulder relative to the reference method is clear. Both in removing Gaoss noise and salt and pepper noise, has a good effect. Can be seen from Table 1, this denoising method under peak high signal noise ratio, is better than the wavelet transform.

\section{Conclusion}

This paper proposes a denoising method based on support vector machine and the simulation experiment of the algorithm. The focus of this chapter is on the selection of support vector machine (SVM) eigenvector adopted a new method, first the image wavelet decomposition to get the wavelet coefficients in all directions, the coefficient of threshold 01 normalized processing yes coefficient, and then by choosing qualified neighborhood average amplitude of wavelet coefficient values as a feature vector. Based on support vector machine of training, the support vector machine model is used to distinguish between noise and the original image, to achieve the effect of denoising. The experimental results show that the method can well remove the noise, and can save images of some important details, with other denoising method based on wavelet transform is compared, has a good advantage

\section{References}

[1]. Q. Pan, L. Zhang and G. Dai, et al., "Two denoising methods by wavelet transform", IEEE Transactions on Signal Processing, vol. 47, no. 12, (2009), pp. 3401-3406.

[2]. S. Tong and D. Koller, "Support vector machine active learning with applications to text classification", The Journal of Machine Learning Research, vol. 2, (2006), pp. 45-66.

[3]. S. Tong and E. Chang, "Support vector machine active learning for image retrieval", Proceedings of the ninth ACM international conference on Multimedia, ACM, (2010), pp. 107-118.

[4]. I. Tsochantaridis, T. Hofmann and T. Joachims, et al., "Support vector machine learning for interdependent and structured output spaces", Proceedings of the twenty-first international conference on Machine learning, ACM, (2004), pp. 104.

[5]. S. Abbasion, A. Rafsanjani and A. Farshidianfar, et al., "Rolling element bearings multi-fault classification based on the wavelet denoising and support vector machine", Mechanical Systems and Signal Processing, vol. 21, no. 7, (2007), pp. 2933-2945.

[6]. A. Buades,B. Coll and J. M. Morel, "A review of image denoising algorithms, with a new one", Multiscale Modeling \& Simulation, vol. 4, no. 2, (2005), pp. 490-530.

[7]. B. Y. Sun, D. S. Huang and H. T. Fang, "Lidar signal denoising using least-squares support vector machine", IEEE Signal processing letters, vol. 12, no. 2, (2005), pp. 101-104.

[8]. M. Antonini, M. Barlaud P. Mathieu, et al., "Image coding using wavelet transform', Image Processing, IEEE Transactions on, vol. 1, no. 2, (2012), pp. 205-220.

[9]. J. A. K. Suykens and J. Vandewalle, "Least squares support vector machine classifiers", Neural processing letters, vol. 9, no. 3, (2009), pp. 293-300.

[10].K. R. Coombes, S. Tsavachidis and J. S. Morris, et al., "Improved peak detection and quantification of mass spectrometry data acquired from surface- enhanced laser desorption and ionization by denoising spectra with the undecimated discrete wavelet transform", Proteomics, vol. 5, no.16, (2005), pp. 41074117.

[11].K. Dabov, A. Foi and V. Katkovnik, et al., "Image denoising by sparse 3-D transform-domain collaborative filtering", Image Processing, IEEE Transactions on, vol. 16, no. 8, (2007), pp. 2080-2095.

[12].M. Alfaouri and K. Daqrouq, "ECG Signal Denoising By Wavelet Transform Thresholding", American Journal of applied sciences, vol. 5, no. 3, (2008).

[13].L. Pasti, B. Walczak and D. L. Massart, et al., "Optimization of signal denoising in discrete wavelet transform", Chemometrics and intelligent laboratory systems, vol. 48, no. 1, (2009), pp. 21-34.

[14].J. L. Starck, E. J. Candès and D. L. Donoho, "The curvelet transform for image denoising", Image Processing, IEEE Transactions on, vol. 11, no. 6, (2002), pp. 670-684. 


\section{Authors}

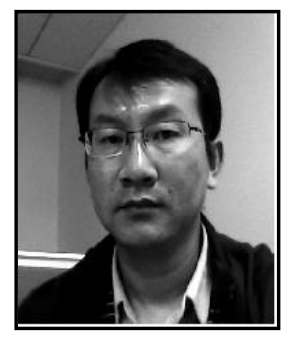

Xinfeng Guo graduated from shanxi university in 1999 and had a b.s.degree. He received Master of Engineering from Shanxi University in 2010. He is a teacher in Engineering College of Shanxi University since 1999.He was studying Knowledge-Discovery in Databases and Web resource discovery. His current research interests on Image noise algorithm and Automation requirements modeling.

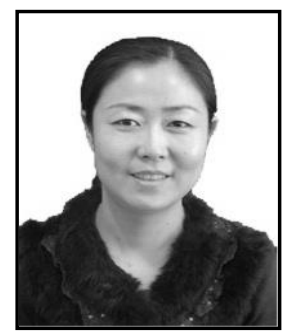

Chunyan Meng, Female, associate professor, was born in Taiyuan of Shanxi Province in 1971. Her research area is computer network technology and computer application technology. 